\title{
Significant Coronary Artery Disease in Patients with a Negative Calcium Scan and Suspicious Ischemic Heart Disease in a Racially Diverse Patient Population
}

\author{
Tara Jarreau, Sargis Khoobiar, Sampoornima Setty, Marc Cohen and Chunguang Chen*
}

Section of Cardiac Imaging, Division of Cardiology, Newark Beth Israel Medical Center, Newark, Newark, New Jersey, USA

\begin{abstract}
Background: Although coronary calcium assessment has been demonstrated to be strongly associated with significant coronary artery disease (CAD) and future cardiac events, it is still not clear whether zero calcium on CT scan can exclude significant CAD and eliminate the need for further multi-detector computed tomography (MDCT) coronary angiography (CCTA). The purpose of this study is to test the hypothesis that zero coronary calcium on MDCT does not exclude significant CAD and CCTA is required to exclude CAD in diverse populations who have a zero calcium score.

Methods: We studied 737 consecutive patients, 383 females and 354 males (average age $57.4+/-12.9$ years), referred to CCTA from June 1, 2005 to January 31, 2007 for clinically suspicious CAD. All patients underwent 16-slice MDCT $(n=287)$ or 64 -slice MDCT $(n=450)$ calcium score scan prior to CCTA. We analyzed atherosclerotic plaques and degree of coronary artery stenosis on CCTA, and coronary calcium score calculated on MDCT calcium scan. Significant CAD was defined by $>50 \%$ coronary artery diameter stenosis.

Results: Significant CAD was found in 211 of 737(29.6\%) patients. Of these patients, $186(88.2 \%)$ patients had a positive calcium score and $25(11.8 \%)$ patients had a zero calcium score. Significant CAD in patients with zero calcium score was found more often in younger $(\mathrm{p}<0.01)$ and female patients $(\mathrm{p}<0.05)$. There is no significant racial difference $(\mathrm{p}=\mathrm{NS})$ in patients with significant $\mathrm{CAD}$ without a positive calcium score.

Conclusion: Absence of coronary calcium deposit does not exclude the presence of significant CAD with non-calcified plaque. Therefore, a calcium score of zero on MDCT calcium scan does not preclude the need for MDCT coronary angiography in patients with clinically suspicious CAD.
\end{abstract}

Keywords: Zero calcium score, coronary artery stenosis, CT coronary angiography.

\section{BACKGROUND AND INTRODUCTION}

Age- and sex-specific calcium score percentiles have been demonstrated to be a significant predictor for coronary events and incrementally added to the prognostic value of traditional risk factors for coronary artery disease (CAD)[1, 2]. However, the incidence of significant CAD, and subsequent cardiac events in patient with a zero coronary calcium score are not negligible and vary from $<1 \%-16 \%$ during the follow-up period (up to 10 years) [3-5]. Furthermore, a limited number of published data on computer tomography (CT) coronary angiography has shown that significant CAD was found in a significant portion of patients with no or minimal coronary calcium, particularly in patients with chest pain or clinical suspicion of ischemic heart disease [3-7]. However, these studies included mostly Caucasian populations [3-10]. Since significant difference in association of coronary calcium with significant coronary disease has been reported in patients with different races (MESA) [11], incidence of significant coronary artery stenosis is still unknown in non-caucasian

*Address correspondence to this author at the Cardiac Imaging and Cardiac Non-Invasive Laboratory, Newark Beth Israel Medical Center, 201 Lyons Avenue, Newark, NJ 07112, USA; Tel: 973-926-7475; Fax: 973-318-7207; E-mail: cchen@sbhcs.com patients who have no detectable calcium on CT calcium scan. Consequently, it is still not clear whether patients referred for suspicion of CAD, who have no calcium on multi-detector CT (MDCT) scan should undergo coronary CT angiography (CCTA) to exclude significant coronary artery stenosis.

The purposes of the present study are to examine the incidence of significant CAD in a diverse patient cohort with different races, ages and gender who underwent CCTA, and to test the hypothesis that zero coronary calcium score on MDCT calcium scan does not exclude significant coronary artery disease and CT coronary angiogram is required to exclude coronary artery disease even in clinically suspicious patients with a zero or minimal calcium score.

\section{PATIENTS AND METHODS}

There were 791 patients referred for CCTA during June 1, 2005 to January 31, 2007 for clinically suspicious CAD with chest pain, abnormal stress test or combination of both with intermediate or low probability of CAD. Of these patients, 6 patients were excluded due to severe renal dysfunction (GFR $<15 \mathrm{~mL} / \mathrm{min}$ ) without dialysis. CCTA was performed in 785 patients, 48 patients were excluded with either inadequate imaging quality for interpretation of CCTA in 13 patients with motion artifacts or unable to hold 
breath, significant arrhythmia with atrial fibrillation or frequent premature beats in 5 patients, inability to quantify calcium score in 16 patients with coronary stent implantation or coronary bypass surgery with metal clips at the anastomosis site in 14 patients. There were 737 patients (Table 1) who were included in this study. Patients with ST segment elevation were not considered with an indication for CCTA at Newark Beth Israel Medical Center and were not studied.

\section{CT Coronary Angiography and Pre-Test Preparation}

Beta-blocker (IV or oral) or calcium channel blocker (if patient had contraindications to beta-blockade) was used if heart rate $>60$ BPM unless a history of severe left ventricular or right ventricular dysfunction or decompensated congestive heart failure, or blood pressure $<95 / 60 \mathrm{mmHg}$ was present. Sublingual nitroglycerin (NTG, $0.4 \mathrm{mg}$ ) was given routinely to dilate coronary arteries and to optimize imaging quality if there were no contraindications (severe aortic valve stenosis, IHSS, allergy to NTG, BP $<95 / 60 \mathrm{mmHg}$, or on medications such as sildenafil). Anaphylactic allergy to contrast agent was considered a contraindication to CT coronary angiography. Pre-medication was used in patients with nonanaphylactic contrast allergy or in patients with allergies to shellfish. Pre-medication included three agents IV (solumedrol $120 \mathrm{mg}$, benadryl $50 \mathrm{mg}$, and pepcid $20 \mathrm{mg}$ ) which were given at least 15 minutes before cardiac CT scan. Metformin was withhold for 48 hours before and after contrast administration in all patients. Patients were assessed for risk of contrast induced nephropathy (CIN) based on GFR rather than on the absolute level of serum creatinine. GFR > $60 \mathrm{~mL} / \mathrm{min}$ : normal or near-normal renal function and extremely low risk for CIN. These patients required no specific prophylaxis or oral hydration was given in patients with multiple risk factors. GFR of 30 to $60 \mathrm{~mL} / \mathrm{min}$ : moderate renal dysfunction and low-to-moderate risk for CIN, IV dyhration of $1000 \mathrm{~mL} \mathrm{D5W}$ with $3 \mathrm{~mL} / \mathrm{kg} / \mathrm{h}$ for 1 hour prior to contrast administration and $2 \mathrm{~mL} / \mathrm{kg} / \mathrm{h}$ after contrast administration; GFR $<30 \mathrm{~mL} / \mathrm{min}$ : severe renal dysfunction and high risk for CIN, hydration and IV sodium bicarbonate regimen was used with $\mathrm{NaHCO}_{3}, 3$ amps (150 $\mathrm{mEq}$ ) in $850 \mathrm{~mL} \mathrm{D} 5 \mathrm{~W}$ at $3 \mathrm{~mL} / \mathrm{kg} / \mathrm{h}$ for 1 hour before contrast administration and at $2 \mathrm{~mL} / \mathrm{kg} / \mathrm{h}$ after contrast administration; GFR $<15 \mathrm{~mL} / \mathrm{min}$ : renal failure. These patients were usually required to be on dialysis and no CCTA with contrast was performed without dialysis or backup of dialysis if renal failure deteriorated.

\section{Acquisition of Cardiac CT Images}

Anterior-posterior and lateral chest scout views were first obtained for planning. Calcium score images were obtained using 64 x $2.5 \mathrm{~mm}$ x $400 \mathrm{~ms}$ with $120 \mathrm{kVp}$ and 50-75 mAs to cover the entire heart and proximal ascending aorta. A positive calcium score was defined by $130 \mathrm{HU}$ with an area of $1 \mathrm{~mm}^{2}$ or greater. The amount of calcium was quantified using the Agatston scoring method [12].

Table 1. Baseline Demographic Characteristics of Patient Population $(n=737)$

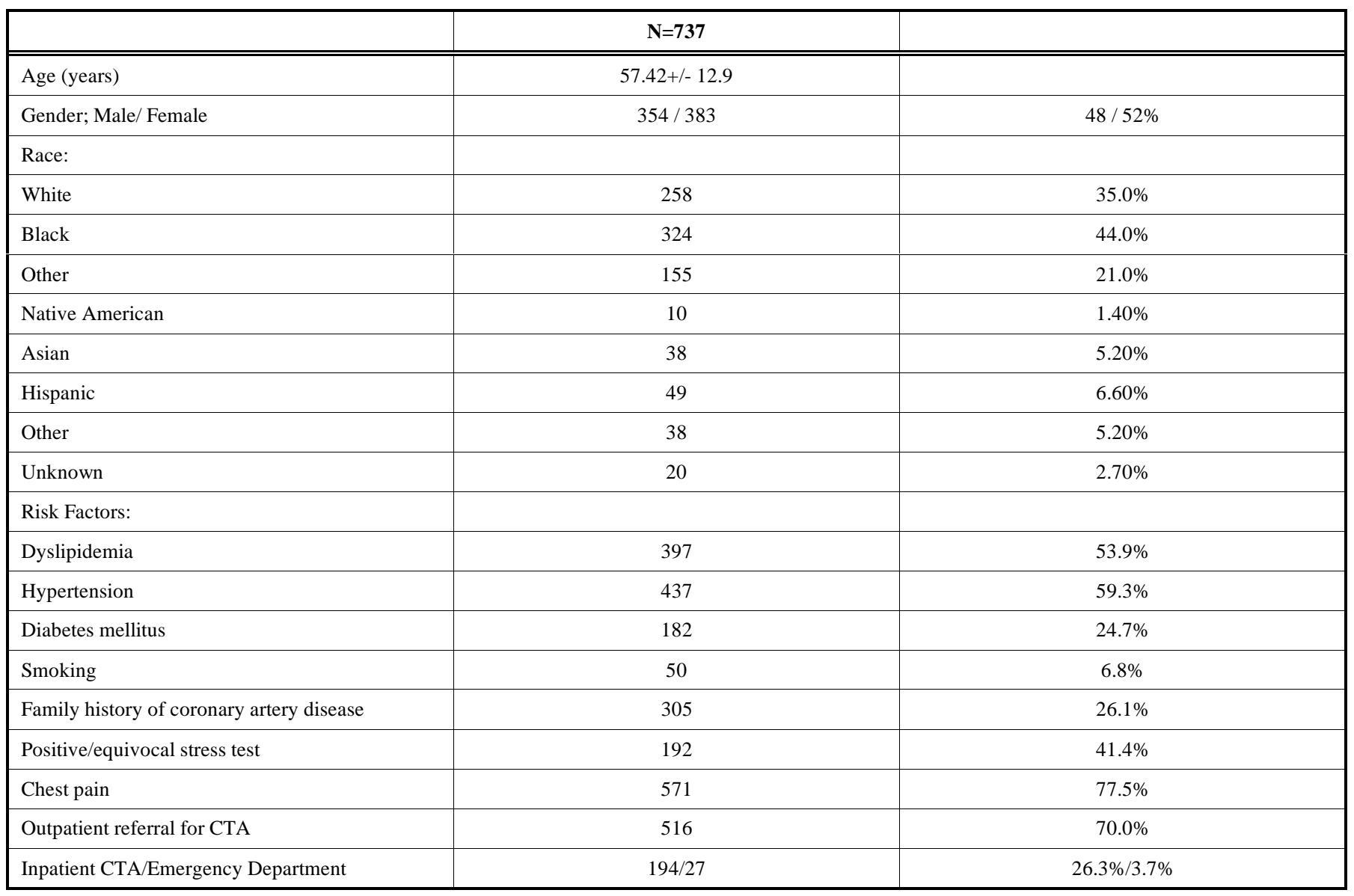


CCTA data were obtained using the thinnest slice thickness and fastest rotation $(0.6 \mathrm{~mm} \times 64 \times 400 \mathrm{~ms})$ for Phillips 64 MDCT scanner and $(0.625$ x $16 \quad \mathrm{x}$ $350 \mathrm{~ms} /$ rotation) for GE, Lightspeed 16 MDCT scanner. A pitch of 0.25 was used. Tube current of 750-1050 mAs/slice (350-450 mA) was selected for CCTA. Higher tube current was used for obese patients. Tube voltage of $120 \mathrm{kVp}$ was routinely used for CCTA scan. In obese patients (more than $300 \mathrm{lbs}$ ), a higher tube voltage of $140 \mathrm{kV}$ was used. The right antecubital vein was usually the preferred site for contrast injection with an 18 gauge IV line. Dual injector was used for injection of contrast (Visipaque) and chasing (70\% saline and $30 \%$ contrast mixture) solution. Contrast injection rate was 4.5-5.5 $\mathrm{ml}$ depending on patient body surface area, heart size and function. A higher rate was used for patient with an enlarged heart and poor LV function. A contrast dose of 70$90 \mathrm{ml}$ of Visipaque was given depending on scan length and time: (total scanning time $\mathrm{x}$ injection flow rate) + (bolus tracking time delay to the start of CT scan $\mathrm{x}$ injection flow rate). The mixture of saline and contrast in the chasing solution is to ensure visualization of RV cavity but not to overshadow RCA visualization.

Bolus tracking method was used for timing of the start of scanning after beginning of the contrast injection. The ascending aorta at bifurcation of pulmonary artery was used as bolus tracking locator site. The threshold for CT unit was set to $140 \mathrm{HU}$ to trigger the scan with a 5-6 second delay. All calcium and cardiac CT scans were reviewed by an experienced cardiologist (CC) as a part of routine clinical report. Extracardiac CT findings were reported by radiologists.

\section{Reconstruction}

Raw CTA scan data were retrospectively reconstructed initially at $75 \%$ of R-R interval to coincide with diastasis and $35-45 \%$ to capture isovolumic relaxation time and endsystole where coronary motion is relatively stable. Additional phases with $5 \%$ increments were reconstructed such as $70 \%-80 \%$, if motion artifacts were noted from the $75 \%$ data. Adaptive cardiac multicycle (or multi-segment) algorithms were used to combine data from multiple cardiac cycles (up to 4) to optimize temporal resolution [13].

\section{CCTA Interpretation}

Source images were reviewed and processed with straightened and curved multi-planar projection reconstruction (MPR), maximal intensity projection (MIP) and cross-sectional views. Measurements of degree of stenosis on CCTA were performed on straighten MPR views and cross-sectional images using Phillips work station. The coronary trees were segmented according to modified American Heart Association classification. The segments were investigated for luminal narrowing. Segments were graded as small (diameter $<1.5 \mathrm{~mm}$ ) which were excluded from analysis. Presence of calcified plaque was defined by any discernible structure in the coronary arterial wall with any focal or dense calcification as defined by a CT density of $\mathrm{HU}$ of $>130$ [14]. Non-calcified plaques were defined as any discernible structure in the coronary arterial wall with a CT density less than the contrast enhanced lumen but different from the surrounding connective tissues without any focal or dense calcification. Degree of coronary artery stenosis was

expressed as \% diameter stenosis. Assessment of degree of stenosis was based on the quantitative measurement and classified as mild $(<30 \%)$, mild to moderate $(30-49 \%)$, moderate $(50-70 \%)$, severe $(>70 \%)$ coronary artery stenosis. A significant coronary artery stenosis was defined as $50 \%$ or greater diameter stenosis. Clinical decision making regarding, medical therapy, further diagnostic testing such as cardiac catheterization and angiography, or revascularization (percutaneous or surgical) was at the discretion of the referring physician.

\section{Statistical Analysis}

The continuous variables were analyzed using one way ANOVA. Non-continuous variables were analyzed with ChiSquare test. Stepwise, multi-variant linear regression analysis was performed to test factors (age, gender, race, hypertension, dyslipidemia, diabetes, family history of premature coronary artery disease, smoking) related to significant coronary artery stenosis with zero calcium score. Numeric data were expressed as mean +/- standard deviation. $\mathrm{P}$ value of 0.05 or less was considered as statistically significant.

\section{RESULTS}

Of 737 consecutive patients included in this study, there were 383 females and 354 males with age of $57.4+/-12.9$ (range 26-89) years. The patient population included $44 \%$ African Americans, 35\% Caucasians, $21 \%$ other races $(6.6 \%$ Hispanics, 5.2\% Asians, and 1.7\% Native Americans, $7.5 \%$ undefined). There were 516 outpatients, and 221 inpatients or patients admitted through the emergency room (See Table 1). There were 315 patients who had chest pain syndrome, 171 patients who had equivocal/positive stress test with dyspnea or palpitations and 251 patients had both of chest pain and equivocal/positive stress test.

Of 737 patients, there were 351 patients with zero calcium score and 386 patients with a positive calcium score. In 386 patients with a positive calcium score, 170 patients were white and 216 patients were non-white. The prevalence of positive calcium score was $65.9 \%$ (170/258 patients) for white patients which was significantly higher $(\mathrm{p}<0.01)$ than non-white patients $(45.1 \%, 216 / 479$ patients). There was no significant difference $(\mathrm{p}=\mathrm{NS})$ in age $(57.3+/-14.4$ vs. 56.6 +/- 13.1 years white vs. non-white) or gender (female/male ratio $163 / 121$ vs. $270 / 232$ patients for white vs. non-white) distribution in white and non-white patients.

A significant coronary artery stenosis was found in 211 of the 737 patients $(28.6 \%), 88 / 258$ (34.1\%) patients for white and 122/479 $(25.5 \%)$ patients for non-white $(\mathrm{p}<0.05)$. Of these 211 patients with significant CAD, 186 patients $88.2 \%$ had positive calcium scores Fig. (1A), and 25 (11.8\%) had zero calcium scores in spite of significant coronary stenosis Fig. (1B) $(\mathrm{p}<0.01)$ (See Table 2). The latter patients had obstructive coronary artery disease with non-calcified plaques, and thus, were not detected by MDCT calcium scan prior to coronary CT angiography.

The prevalence of significant coronary artery stenosis is $48.3 \%$ (186/385 patients) in patients with a positive calcium score in this study population with a clinically suspicious CAD. There was no significant difference between Caucasian patients with a positive calcium score and 


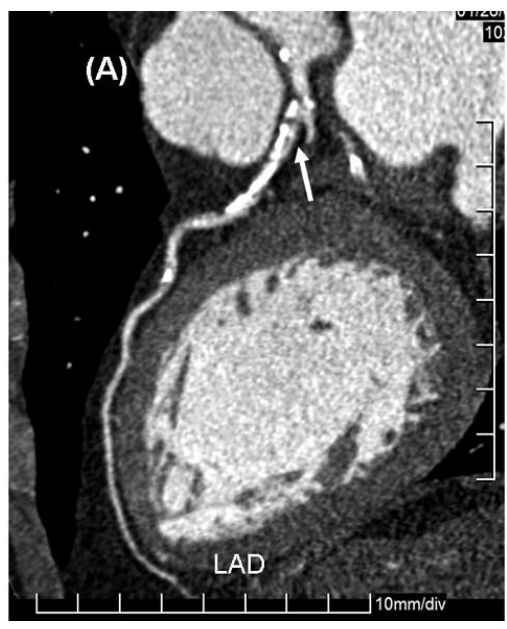

Fig. (1). A: MDCT coronary angiography shows calcified plaque with significant LAD stenosis (arrow). Note that there is also significant left main stenosis.

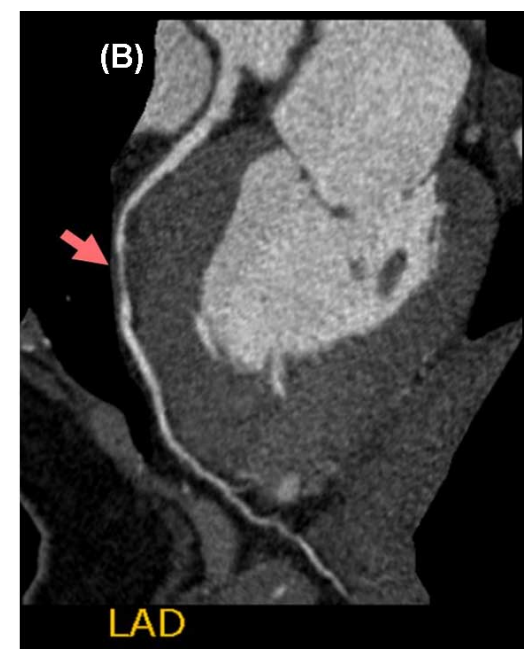

Fig. (1). B: MDCT coronary angiography shows non-calcified plaque with significant LAD stenosis (arrow) after sublingual nitroglycerine.

significant CAD $(81 / 170$ patients, $47.6 \%)$ and nonCaucasian patients $(105 / 216$ patients, $48.6 \%)$. In patients with a positive calcium score on MDCT calcium scan and significant coronary artery stenosis, the majority of patients $(60.2 \%$, Table 3$)$ had a calcium core of 400 or more. Of note, $12.3 \%$ of patients with a significant coronary artery stenosis had a minimal calcium score of $<100$ but $>0$.

From the 526 patients without significant stenosis on CCTA, there were 326 patients who had zero calcium scores and 199 patients had a positive calcium score. Of 326 patients with zero calcium score, there were 80 white patients and 246 non-white patients.

The prevalence of significant CAD was $7.1 \%(25 / 351$ patients) in patients with a zero calcium score in this study. There was no significant difference $(\mathrm{p}=\mathrm{NS})$ between the prevalence of significant CAD for white patients $(9.1 \%, 8 / 88$ patients) and for non-white patients $(6.5 \%, 17 / 263$ patients) with a zero calcium score. There was no difference of the prevalence of significant coronary artery stenosis between 63 of $221(28.5 \%)$ inpatients or emergency department patients, and 148 of $516(28.7 \%)$ outpatients $(\mathrm{p}=\mathrm{NS})$. Duration of chest pain of significant CAD with positive calcium $(2.5+/-$ 1.8 months) was not different from that of significant CAD patients with negative calcium $(2.2+/-2.1$ months, $\mathrm{p}=\mathrm{NS})$. There was tendency toward a higher proportion of a significant coronary artery stenosis without calcified plaque in non-Caucasian patients $(13.9 \%, 17 / 122$ patients) with significant CAD than that of Caucasian $(9 \%, 8 / 89)$ patients with significant $\mathrm{CAD}$ but it did not reach statistical significance ( $p=N S$ ). Traditional coronary risk factors were compared between the two groups of patients with significant coronary stenosis, with zero calcium scores versus those with positive calcium scores, using stepwise multivariate linear regression analysis. The only two factors that reached statistical significance were age and female gender. Patients with calcium scores of zero were younger $(\mathrm{p}<0.01)$ than those with positive calcium score. There was a wide range of age in patients with zero calcium scores and significant CAD (26-80 years) as compared to those with positive calcium scores (44-89 years). In 25 patients with zero calcium scores and significant CAD, 18 (72\%) patients were 50 years or older and 9/25 (36\%) patients were 60 years or older. In 25 patients with zero calcium score and significant coronary artery stenosis, $15(60 \%)$ patients were

Table 2. Factors Related to Calcium Score in Patients with Significant Stenosis $(n=211)$

\begin{tabular}{|c|c|c|c|c|}
\hline & & Calcium $=0(n=25)$ & Calcium > $0(n=186)$ & P-value \\
\hline Race: & White & $8(32 \%)$ & $81(43.5 \%)$ & NS \\
\hline \multicolumn{2}{|c|}{ Women } & $15(60 \%)$ & $79(42 \%)$ & $<0.05$ \\
\hline \multicolumn{2}{|c|}{ Hypertension } & $16(64 \%)$ & $120(64.5 \%)$ & NS \\
\hline \multicolumn{2}{|c|}{ Current smoker } & $3(12 \%)$ & $12(6.5 \%)$ & NS \\
\hline \multicolumn{2}{|c|}{ Hyperlipidemia } & $15(60 \%)$ & $121(65.1 \%)$ & NS \\
\hline \multicolumn{2}{|c|}{ Family history of CAD } & $12(48 \%)$ & $83(44.6 \%)$ & NS \\
\hline \multicolumn{2}{|c|}{ Chest pain } & $15(60 \%)$ & $129(69.4 \%)$ & NS \\
\hline
\end{tabular}


Table 3. Quartile Quantitative Calcium Score in Patients with Significant CAD

\begin{tabular}{|c|c|c|}
\hline Quantitative Calcium Score & Patients & \% \\
\hline \hline$* 0$ & 25 & $11.8 \%$ \\
\hline$<100$ & 26 & $12.3 \%$ \\
\hline $100-199$ & 15 & $7.1 \%$ \\
\hline $200-399$ & 33 & $8.5 \%$ \\
\hline $400-1000$ & 94 & $15.6 \%$ \\
\hline$>1000$ & 211 & $44.6 \%$ \\
\hline Total & $100 \%$ \\
\hline
\end{tabular}

female as compared only 79/186 (42\%) patients with positive calcium score and significant coronary artery stenosis were female $(\mathrm{p}<0.05)$. Other factors including race, hypertension, diabetes, current smoking status, hyperlipidemia, family history of coronary artery disease, inpatients or outpatient population or the presence of chest pain were not predictive of significant non-calcified lesions (Table 2).

Comparison between CT coronary angiography and cardiac catheterization angiography: For the entire group, there were 43 patients who had invasive coronary angiography within 3 months of CT angiography and without interval events. 39 of these 43 patients, had significant coronary artery stenosis on invasive cardiac catheterization coronary angiography; 37 of the 39 patients had significant coronary artery stenosis $(\geq 50 \%)$ on $\mathrm{CT}$ coronary angiography (sensitivity of 95\%). Only two patients with $>50 \%$ coronary artery stenosis on catheterization were slightly underestimated by CT $(25-49 \%$ coronary stenosis by CT). The remaining 4 of the 43 patients, did not have a significant coronary artery stenosis on CT, nor on cardiac catheterization. This was too few to calculate specificity. Of 25 patients with significant coronary artery stenosis with zero calcium score on CT, $18(72 \%)$ patients underwent cardiac catheterization and were all confirmed by invasive coronary angiography to have a significant coronary artery stenosis Fig. (1B and $\mathbf{C})$. Thirteen of the $18(72 \%)$ patients with significant CAD with zero calcium score who had catheterization coronary angiography underwent percutaneous coronary intervention $(n=11)$ with stents or coronary bypass surgery $(n=2)$. The remaining patients were treated medically.

\section{DISCUSSION}

The main findings of this study are: 1) A significant number $(11.8 \%)$ of patients with significant coronary artery stenosis on CT coronary angiography have a zero calcium score on CT calcium scan in a large, diverse, mostly nonCaucasian patient population, 2) There is no significant racial difference in incidence of significant CAD with zero calcium score in Caucasian and non-Caucasian patients, 3) Significant CAD without detectable calcium deposit is more common in younger patients but can occur in as old as 80 years old, 4) Females are more likely to have significant coronary artery stenosis without positive calcium scan than males. Thus, a negative calcium scan does not exclude significant coronary artery disease, particularly in younger and female patients either in Caucasian or non-Caucasian population. 5) Significant CAD with non-calcified plaques is not benign as more than $70 \%$ patients with non-calcified plaques required percutaneous or surgical coronary intervention. Therefore, CT coronary angiography should be performed even in patients with no detectable calcium deposit on the coronary system to exclude significant coronary artery stenosis in clinically suspicious CAD.

\section{CALCIUM SCORE AND SIGNIFICANT CAD}

Calcium scores of greater than $100 \mathrm{HU}$ have been described as an independent risk factor for coronary artery disease [15]. Previous studies show inconsistent results regarding prevalence of patients with significant CAD without a positive calcium score. Significant CAD with zero calcium score varied from negligible of $<1 \%$ to $16 \%$ of total patients with significant CAD. The difference may be caused by differences in patient selection (symptomatic or asymptomatic screening population, racial variations), duration of follow-up and definition of significant CAD. Difference in prevalence of significant positive calcium deposit in the coronary artery system between Caucasian and

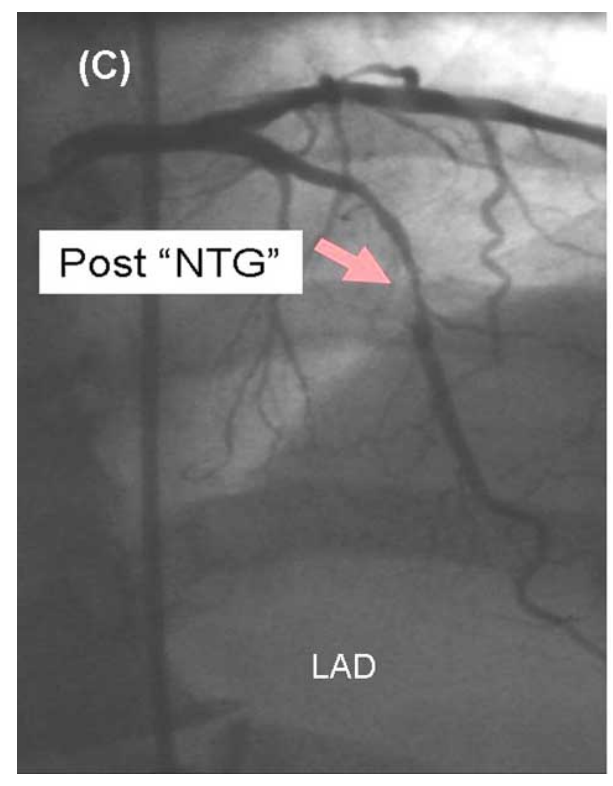

Fig. (1). C: The corresponding invasive coronary angiogram for significant LAD stenosis (arrow) with non-calcified plaque of B on CT coronary angiogram after intracoronary nitroglycerine to exclude coronary spasm. 
non-Caucasian was demonstrated by Detrano et al. [16]. Whether or not the racial difference may contribute to the discrepancy in prevalence of significant CAD without calcium in previous studies was not clear. In a diverse population consisting of primarily Africa-Americans, we demonstrated in this study that the majority of patients with a significant coronary artery stenosis had a calcium score of 100 or more $(76 \%)$. Furthermore, $44 \%$ of patients with significant coronary artery stenosis had a calcium score of 1000 or more. However, there was $24.1 \%$ of patients with significant coronary artery stenosis who had a calcium score less than 100. In fact, $11.8 \%$ patients with significant coronary artery stenosis had no detectable calcium deposit in the coronary artery at all. Patients with significant coronary disease and zero coronary calcium are generally younger and more likely to be female than those with detectable calcium deposit in the coronary artery. The difference in average ages is expected as calcification is more prevalent in older adults and plaques are more likely to calcify over time [17]. However, there was a wide range of age in patients with zero calcium scores and significant CAD (26-80 years) as compared to those with positive calcium scores (44-89 years). In fact, 18/25 (72\%) of patients with zero calcium scores and significant CAD were 50 years or older and $9 / 25$ $(36 \%)$ were 60 years or older. This demonstrates that a zero calcium score can not be used to rule out significant coronary artery disease even in the older population of patients of age of 60 years or older. The reason for a significant higher incidence in women than men with significant coronary disease without detectable calcium in the coronary system is not clear [5]. Whether it reflects late occurrence of coronary artery disease after 55 years old in females or female hormones that influence the composites of coronary artery atherosclerotic plaques would need further study [18]. Non-Caucasian patients have a tendency toward a slightly higher proportion of significant CAD with noncalcified plaque and zero calcium score than white patients. However, the differences did not reach a statistical significance $(\mathrm{p}=\mathrm{NS})$. Caucasian had both a higher incidence of significant $\mathrm{CAD}$ and a higher incidence of positive calcium scan in this study while proportion of patients with significant CAD who had a positive calcium scan is not different between Caucasian (47.6\%) and non-caucasian $(48.6 \%)$ patents. The tendency of a higher calcium score in Caucasian patients is consistent with results of Detrano et al's study in which prevalence of coronary calcification (calcium score $>0$ ) is higher in white men $(70.4 \%)$ or white women $(44.7 \%)$ than black men $(56.6 \%)$ or women $(37 \%)$, Hispanic men $(56.6 \%)$ or women $(34.8 \%)$, or Chinese men $(59.2 \%)$ or women $(41.9 \%)$; however, predictive value of calcium score for coronary events was not different between white and non-white races [16].

\section{COMPARED WITH PREVIOUE STUDIES}

The high percentage of patients with significant CAD without positive calcium scan in this study is consistent with results of Schenker et al. [4] and Hausleiter et al. [19]. In the study of Hausleiter et al., 10 of 108 (9.3\%) patients with significant CAD with a zero calcium score in patients with clinically suspicious CAD either with chest pain, significant arrhythmia or positive stress test. In the study of Schenker et al., the incidence of ischemia on PET was $16 \%$ of patients with no calcium on MDCT calcium scan. Although the racial distribution is not clear in their study, patients included in their study were similar to our study and were all-physician referred for evaluation of CAD based on cardiac symptoms or other evidence of intermediate-to-high risk of CAD. In contrast, in prior studies which reported low incidence of significant $\mathrm{CAD}$ without positive cardiac scan, patients were largely screening populations with or without cardiac symptom [17], or symptomatic patients with high probability of CAD were referred for cardiac catheterization in mostly Caucasian patients by Knez et al. and Budoff et al. [5, 20].

\section{RATIONALE OF NON-CALCIFIED PLAQUES FOR OBSTRUCTIVE CAD}

The cause of significant $\mathrm{CAD}$ without a positive calcium scan is unclear. It may be related to limitations of current calcium CT scan and various compositions of different stages of atherosclerotic plaques. Histopathological plaque characteristic studies demonstrated that microcalcifications can be seen in various stages of the atherosclerotic disease [21]. The calcifications are dense and become identifiable on CT scans in more advanced stage of atherosclerotic plaques. At least $1 \mathrm{~mm}^{2}$ of calcium deposit or 3 pixels of calcium density is required to identify calcium on CT scans. Although calcium score correlates linearly with total plaque area on histopathological studies by Rumberger et al. [15], it was noted that calcified plaques only account for $20 \%$ of the total plaque burden. The vast majority of plaques are noncalcified at early stages of plaque formation and may not necessarily be obstructive. Furthermore, the eroded plaques seen in pre-menopausal women have a much lower incidence of calcification [22].

\section{LIMITATIONS}

While our diverse patient population with coronary CTA and calcium scores is one of the largest series of patients in the literature, we recognize that there are limitations in the present study. This study is retrospective. Patients included in this study were referred to coronary CTA with selection bias by referring physician. However, all patients were clinically suspicious for significant CAD. The majority of our patients suffered from chest pain and many also had abnormal or equivocal stress testing. Not all patients underwent an exercise or pharmacological stress test either pre-CCTA or post CCTA in this study, data might have looked differently if all patients had underwent an exercise stress test.

\section{CONCLUSION}

A negative calcium scan does not preclude the need for CT coronary angiography in patients with clinically suspicious coronary artery disease in Caucasian or nonCaucasian patients. CT coronary angiography provides not only the degree of coronary stenosis in patients with positive calcium scans, but also can detect coronary artery stenosis in patients with negative calcium scans, thus, providing incremental value over calcium scan alone in clinically suspicious CAD patients even with no detectable calcium deposit in the coronary artery. 


\section{DISCLOSURE}

There is no interest of conflict to disclose by any of authors in this study.

\section{REFERENCES}

[1] Greenland P, LaBree L, Azen SP, Doherty TM, Detrano RC. Coronary artery calcium score combined with Framingham score for risk prediction in asymptomatic individuals. JAMA 2004; 291 : 210-5.

[2] LinksWong ND, Budoff MJ, Pio J, Detrano RC. Coronary calcium and cardiovascular event risk: evaluation by age- and sex-specific quartiles. Am Heart J 2002; 143: 456-9.

[3] Hopkins PN, Ellison RC, Province MA, et al. Association of coronary artery calcified plaque with clinical coronary artery disease in the national heart, lung and blood institute's family heart study. Am J Cardiol 2006; 97: 1564-69.

[4] Schenker MP, Dorbala S, Hong ECT, et al. Interrelation of coronary calcification, myocardial ischemia and outcomes in patients with intermediate likelihood of coronary artery disease: a combined positron emission tomography/computed tomography study. Circulation 2008; 117: 1693-1700.

[5] Knez A, Becker A, Leber A, et al. Relation of coronary calcium scores by electron beam tomography to obstructive disease in 2115 symptomatic patients. Am J Cardiol 2004; 93: 1150-2.

[6] Cheng VY, Lepor NE, Madyoon H, Eshaghian S, Naraghi AL, Shah PK. Presence and severity of noncalcified coronary plaque on 64-slice computed tomographic coronary angiography in patients with zero and low coronary artery calcium. Am J Cardiol 2007; 99: 1183-6.

[7] Berman DS, Achenbach S, Taylor AJ, Weigold G, PoonM. Highlights of the Second Annual Scientific Meeting of the Society of Cardiovascular Computed Tomography: Washington, DC, July 6-8, 2007. J Am Coll Cardiol 2007; 50: 2329-35.

[8] LaMonte MJ, Fitzgerald SJ, Church TS, et al. Coronary artery calcium score and coronary heart disease events in a large cohort of asymptomatic men and women. Am J Epidemiol 2005; 162: 421-9.

[9] Kondos GT, Hoff JA, Sevrukov A, et al. Electron-beam tomography coronary artery calcium and cardiac events: a 37month follow-up of 5635 initially asymptomatic low- to intermediate-risk adults. Circulation 2003; 107: 2571-6.

[10] Taylor AJ, Bindeman J, Feuerstein I, Cao F, Brazaitis M, O'Malley PG. Coronary calcium independently predicts incident premature coronary heart disease over measured cardiovascular risk factors: mean three-year outcomes in the Prospective Army Coronary Calcium (PACC) project. J Am Coll Cardiol 2005; 46: 807-14.

[11] Links Bild DE, Detrano R, Peterson D, et al. Ethnic differences in coronary calcification: the Multi-Ethnic Study of Atherosclerosis (MESA). Circulation 2005; 111: 1313-200.

[12] Agatston A, Janowiz WR, Hildner FJ, Zusmer NR, Viamonte M Jr, Detrano R. Quantification of coronary artery calcium using ultrafast computed tomography. J Am Coll Cardiol 1990: 15: 82732.

[13] Manzkle R, Grass M, Nelson T, Shechter G Hawke D. Adaptive temporal resolution optimization in helical cardiac cone beam CT reconstruction. Med Phys 2003; 30: 3072-80.

[14] Detrano CR, Anderson M, Nelson J, et al. Coronary calcium measurements: effect of CT scanner type and calcium measure on rescan reproducibility--MESA study. Radiology 2005; 236: 477-84.

[15] Church T, Levine B, McGuire D, et al. Coronary artery calcium score, risk factors, and incident coronary heart disease events. Atherosclerosis 2003; 190: 224-31.

[16] Detrano R, Gerci AD, Carr JJ, et al. Coronary calcium as a predictor of coronary events in four racial or ethnic groups. N Engl J Med 2008; 358: 13-20.

[17] McClelland RL, Chung H, Detrano R, Post W, Kronmal RA Distribution of coronary artery calcium by race, gender, and age: results from the Multi-Ethnic Study of Atherosclerosis (MESA). Circulation 2006; 113: 30-7

[18] Hodis HN, Mack WJ, Lobo RA, et al. Estrogen therapy and coronary-artery calcification. N Engl J Med 2007; 357: 1252-4.

[19] Hausleiter J, Meyer T, Hadamitzky M, Kastrati A, Martinoff S Schömig A. Prevalence of noncalcified coronary plaques by 64slice computed tomography in patients with an intermediate risk for significant coronary artery disease. J Am Coll Cardiol 2006; 48: 312-8.

[20] Budoff MJ, Raggi P, Arad Y, Guerei AD, Callister TQ, Berman D. Continuous probabilistic prediction of angiographically significant coronary artery disease using electron beam tomography. Circulation 2002; 105: 1791-6.

[21] Rumberger JA, Simons BD, Fitzpatrick LD, Sheedy PF, Schwartz RS. Coronary artery calcium area by electron-beam computed tomography and coronary atherosclerotic plaque area. a histopathologic correlative study. Circulation 1995; 92: 2157-62.

[22] Fuster V, Moreno PR, Fayad ZA, Corti R, Badimon JJ. Atherothrombosis and high-risk plaque. J Am Coll Cardiol 2005; 46: 937-54. 